\title{
COMPORTAMIENTO AGRONÓMICO DE HÍBRIDOS DE MAÍZ EN DOS MUNICIPIOS DE VERACRUZ, MÉXICO ${ }^{1}$
}

\author{
Oscar Hugo Tosquy Valle ${ }^{2}$, Artemio Palafox Caballero ${ }^{2}$, Mauro Sierra Macías $^{2}$, Andrés Zambada Martínez ${ }^{2}$ \\ Rodolfo Martínez Morales ${ }^{3}$, Gonzalo Granados Reinaut 4
}

\section{RESUMEN}

Comportamiento agronómico de híbridos de maíz en dos municipios de Veracruz, México. La productividad del maíz de temporal en Tlalixcoyan y San Andrés Tuxtla, Veracruz, México, está limitada por el uso de genotipos de bajo rendimiento, susceptibles a enfermedades y por la siembra de generaciones avanzadas de variedades mejoradas. Con la finalidad de conocer el comportamiento agronómico de híbridos de maíz en estas localidades, durante el temporal 2002, se evaluaron 23 híbridos experimentales y los testigos regionales $\mathrm{H}$ 520 y H-519C, en un diseño látice 5 x 5 con tres repeticiones. Se midieron las variables: días a floración masculina, altura de planta y mazorca, porcentaje de mazorcas podridas y rendimiento de grano, en ambas localidades y severidad del achaparramiento en Tlalixcoyan. Los resultados indicaron que CMS 993013 y CMS 993015, fueron los híbridos más productivos, con buen comportamiento agronómico y de reacción al achaparramiento en Tlalixcoyan, los cuales superaron en promedio en 3,32 t/ha a los testigos. A su vez, CMS 973023 y CMS 993033 que presentaron bajos porcentajes de pudrición de mazorcas y porte de planta y ciclo intermedio, fueron los mejores híbridos en San Andrés Tuxtla que en promedio superaron en 3,16 t/ha a los testigos. Los mayores rendimientos promedio de localidades se obtuvieron con CMS 973023, CMS 993013 y CMS 993033, aunque sólo el primero y el tercero mantuvieron alto rendimiento en ambas localidades. La correlación mostró que el achaparramiento en Tlalixcoyan y la pudrición de mazorcas en San Andrés Tuxtla afectaron significativamente el rendimiento.

Palabras clave: híbridos, maíz, capacidad productiva, enfermedades.

\begin{abstract}
Agronomic performance of corn hybrids in two municipalities of Veracruz, Mexico. Corn productivity under rainfed, in Tlalixcoyan and San Andrés Tuxtla, Veracruz, Mexico is limited by low yield and disease susceptibility y advanced generations of improved varieties. With the purpose of assessing the agronomic behavior of corn hybrids during the 2002 rainy season, 23 experimental hybrids and the regional varieties $\mathrm{H}-520$ and $\mathrm{H}-519 \mathrm{C}$ were evaluated in the localities indicated above; a 5 x 5 lattice statistical design with three replications was utilized. The variables that were measured in both localities were: days to male flowering, plant and ear height, percentage of rotten ears and grain yield, while the severity to stunting was only measured in Tlalixcoyan. The individual analyses showed significance for all the variables in both localities. CMS 993013 and CMS 993015 were the most productive hybrids with grain yields of 9.57 and $8.44 \mathrm{t} / \mathrm{ha}$, respectively, $3.32 \mathrm{t} / \mathrm{ha}$ higher than the regional varieties. They had good agronomic behavior and were tolerant to the stunting in Tlalixcoyan, CMS 973023 with 7.52 t/ha and CMS 993033 with $7.29 \mathrm{t} / \mathrm{ha}$ were the best hybrids in San Andres Tuxtla; they had an average yield $3.16 \mathrm{t} / \mathrm{ha}$ higher than the regional varieties, presented low percentages of ear rotting and their plant height and life cycle were intermediate. The highest average yields troughout localities were obtained with CMS 973023, CMS 993013 and CMS 993033, which yields were higher than $7.50 \mathrm{t} / \mathrm{ha}$, although only the first and the last one maintained high yield in both localities. The correlation showed that stunting in Tlalixcoyan and ear rotting in San Andrés Tuxtla significantly affected grain yield.
\end{abstract} diseases.

Key words: hybrids, maize, productive capacity,

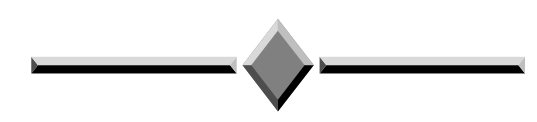

\section{INTRODUCCIÓN}

En México, el maíz es un cultivo de gran importancia, ya que proporciona en promedio $39 \%$ de proteína y el 59\% de la energía que ingieren los mexicanos, como resultado de un consumo per cápita de 209,8 $\mathrm{kg}$ (Morris y López 2000). En el estado de Veracruz, este cultivo se siembra principalmente en temporal, reportándose en el

1 Recibido: 14 de setiembre, 2004. Aceptado: 16 de diciembre, 2004. Presentado en la L Reunión Anual del PCCMCA, El Salvador, 2004.

2 Instituto Nacional de Investigaciones Forestales, Agrícolas y Pecuarias. Campo Experimental Cotaxtla, km 34 carr. Veracruz -Córdoba. Apdo. 429 C.P. 91700 . Medellín de Bravo, Veracruz, México. Email: tosquy.oscar@inifap.gob.mx

3 Consejo de Desarrollo del Papaloapan. Zamora No. 34 zona centro. C.P. 91000. Xalapa, Veracruz, México.

4 Centro Internacional de Mejoramiento de Maíz y Trigo (CIMMYT). Apdo. Postal 6-641 C.P. 06600 México, D. F., México. 
año 2001 una superficie sembrada de 431 mil hectáreas, que representaron el $67 \%$ del total cultivable (INEGI 2002).

Tlalixcoyan y San Andrés Tuxtla son dos municipios maiceros de Veracruz, importantes por su superficie sembrada; sin embargo, su rendimiento promedio es de tan sólo 1,8 t/ha, debido principalmente al uso de generaciones avanzadas de variedades mejoradas en el 70 $\%$ de la superficie (Tosquy et al. 2001), de bajo potencial de rendimiento, susceptibles a pudriciones de la mazorca (Cepeda y Orozco 1989) frecuente en áreas de mayor humedad relativa y nubosidad (Betanzos 2001), al achaparramiento ocasionado por el Spiroplasma kunkelli, el Virus del Enanismo arbustivo del maíz y el Virus del Rayado fino del maíz, cuyo principal vector es la chicharrita Dalbulus maydis (Henríquez y Jeffers 1997; Cano et al. 2000), la cual puede reducir considerablemente el rendimiento al disminuir el tamaño y número de granos de la mazorca y limitar la producción de este cultivo en las áreas tropicales de México (Rocha 1987).

El programa de mejoramiento genético de maíz del CIRGOC - INIFAP ha evaluado híbridos de maíz en condiciones de temporal en el sureste de México. Algunos de estos materiales han mostrado buen comportamiento agronómico, sanidad de mazorca y tolerancia al achaparramiento (Hidalgo et al. 1998) y un potencial de rendimiento a nivel experimental y de validación de 8 y $6 \mathrm{t} / \mathrm{ha}$, respectivamente (Palafox et al. 2001), por lo que se encuentran en proceso de liberación y difusión a los productores. Por otro lado, recientemente este programa en colaboración con el CIMMYT ha evaluado regionalmente un conjunto de híbridos comerciales y experimentales generados para condiciones de trópico húmedo. Como parte de estos trabajos de colaboración y con la participación del Consejo de Desarrollo del Papaloapan (CODEPAP), durante el ciclo primavera-verano 2002 se establecieron dos experimentos de híbridos de maíz, uno en el municipio de Tlalixcoyan y otro en San Andrés Tuxtla, en el estado de Veracruz, respectivamente. El objetivo fue identificar los mejores híbridos, en cuanto a su rendimiento, características agronómicas y de sanidad de cultivo, que en un futuro puedan representar nuevas alternativas tecnológicas para los productores de esas zonas de producción.

\section{MATERIALES Y MÉTODOS}

Durante el ciclo primavera-verano 2002, bajo condiciones de temporal se establecieron dos ensayos de híbridos de maíz previamente seleccionados por su adaptación a las regiones tropicales húmedas del sures- te de México. Estos se ubicaron en el ejido Santa María, en el municipio de Tlalixcoyan y en el ejido Comoapan, San Andrés Tuxtla. El tipo de clima que prevalece en la primera localidad es $\mathrm{Aw}_{2}$, que se clasifica como cálido subhúmedo con lluvias en verano (el más húmedo de los subhúmedos); mientras que en la segunda localidad es Am, cálido húmedo con lluvias en verano y 5 a $10 \%$ de lluvia invernal, de acuerdo a la clasificación de Köppen modificada por García (1981). Los experimentos se establecieron en un tipo de suelo Vertisol, de textura migajón arcillosa para la localidad de Tlalixcoyan y en un Andosol, de textura franca en la localidad de San Andrés Tuxtla, ambos suelos son medios en su contenido de materia orgánica $(2,14$ y 2,23\%, respectivamente) con un $\mathrm{pH}$ de 6,43 y 6,50 considerados ligeramente ácidos (Universidad Autónoma Chapingo 1982; López 1998).

El diseño estadístico utilizado en ambos sitios experimentales fue látice 5 x 5 con tres repeticiones. Se evaluaron 23 híbridos experimentales de maíz de cruza simple, de grano blanco, que han mostrado buen potencial de rendimiento en las zonas tropicales húmedas de México y cuyos progenitores son líneas élite del programa tropical del Centro Internacional de Mejoramiento de Maíz y Trigo (CIMMYT). Los híbridos H-520 y H519C, de cruza triple y también de grano blanco generados por el programa de mejoramiento genético de maíz del Campo Experimental Cotaxtla, perteneciente al Instituto Nacional de Investigaciones Forestales, Agrícolas y Pecuarias (INIFAP), fueron los testigos regionales. Se utilizó una densidad de población de 62,500 plantas por hectárea, con un manejo tecnológico acorde a lo recomendado por el INIFAP para cada región (Tinoco et al. 2002). Las variables medidas en ambas localidades fueron: floración masculina (FM), contando los días a partir de la siembra y hasta que el $50 \%$ de las plantas se encontraron con emisión de polen; altura de planta (AP), desde la base del tallo hasta la inserción de la hoja bandera; altura de mazorca (AM), tomada en centímetros desde la base del tallo hasta el nudo donde se inserta la mazorca principal; por ciento de mazorcas podridas (MP) y rendimiento de grano (RG) ajustado al $14 \%$ de humedad. Así mismo, considerando que en los últimos años en la región donde se ubica la localidad de Tlalixcoyan se ha detectado la presencia de achaparramiento en las siembras comerciales de maíz (Cano et al. 2000), se calificó la severidad de la enfermedad $(\mathrm{ACH})$ con base a la presencia de síntomas de reducción del tamaño de entrenudos, coloraciones rojizas en las hojas, bandas cloróticas en la base de las hojas, proliferación de mazorcas pequeñas, etc., para lo cual se utilizó una escala de uno a cinco, donde 1 correspondió a sano; 2 a presencia de amarillamiento en la base de las hojas inferiores hasta un 25\%; 3 a acortamiento de entrenudos, amarillamiento de la base de las hojas inferiores y en las hojas medias hasta un 
$50 \%$, reducción del tamaño de la mazorca; 4 a enanismo, acortamiento de entrenudos, amarillamiento de las hojas inferiores y hojas medias hasta en un 75\%; 5 a enanismo pronunciado, acortamiento de entrenudos, amarillamiento en un $100 \%$ de las hojas y proliferación de mazorcas pequeñas. La variable mazorcas podridas obtenida en porcentaje fue transformada a $\sqrt{\%}$ de acuerdo con Reyes (1990), aunque para dar mayor claridad a los resultados se presentan los valores reales. Se realizaron análisis de varianza por localidad, para cada una de las variables, utilizando el programa alpha látice (Barreto 1999) y análisis combinado para rendimiento de grano y como prueba de separación de medias se utilizó la Diferencia Mínima Significativa. También se corrieron correlaciones entre las variables mazorcas podridas y severidad del achaparramiento con el rendimiento de grano obtenido por los genotipos para conocer su grado de asociación y si existe efecto significativo entre dichos parámetros (Reyes 1982).

\section{RESULTADOS Y DISCUSIÓN}

Los análisis de varianza individuales en la localidad de Tlalixcoyan, detectaron diferencia altamente significativa en rendimiento de grano, altura de planta y mazorca, días a floración masculina y severidad del achaparramiento y diferencia significativa en el porcentaje de mazorcas podridas. Lo anterior indica el diferente comportamiento que tuvieron los híbridos en esos parámetros. Para el caso de la localidad de San Andrés Tuxtla, existió alta significancia en todas las variables.

El rendimiento y comportamiento agronómico de los híbridos evaluados en Tlalixcoyan se muestran en el Cuadro 1, donde se visualiza que un grupo sobresaliente de 15 híbridos obtuvieron rendimientos superiores a los testigos regionales H-520 y H-519 C (este último híbrido fue el de menor rendimiento), de los cuales CMS 993013, CMS 993015 y CMS 993035 con rendimientos experimentales de 9,$57 ; 8,44$ y $8,08 \mathrm{t} / \mathrm{ha}$, resultaron estadísticamente superiores de acuerdo a la DMS al 1\%, lo que refleja el gran potencial de rendimiento que tienen la mayoría de estos híbridos.

Los resultados señalados concuerdan con lo encontrado por Palafox et al. (2001) que en evaluaciones realizadas bajo condiciones de temporal en varios sitios del sureste de México detectó híbridos de maíz con rendimientos experimentales superiores a $8 \mathrm{t} / \mathrm{ha}$. Con respecto a la altura de planta y mazorca, los valores medios de la mayoría de los híbridos, sugieren un porte de planta y posición de mazorca intermedia (menor de 240 y de $140 \mathrm{~cm}$ para cada variable, respectivamente), ya que en este ciclo la planta alcanza mayor altura y con ello mayor exposición a los vientos. Con base a la prue-
Cuadro 1. Rendimiento de grano y características agronómicas observadas en híbridos de maíz en Tlalixcoyan, Veracruz, México. 2002.

\begin{tabular}{|c|c|c|c|c|c|c|c|}
\hline No. & Genotipo & $\begin{array}{c}\mathbf{R G}^{\mathbf{1}} \\
(\mathbf{t} / \mathbf{h a})\end{array}$ & $\begin{array}{l}\mathbf{A} \mathbf{P}^{2} \\
(\mathrm{~cm})\end{array}$ & $\begin{array}{l}\mathbf{A M}^{\mathbf{3}} \\
(\mathbf{c m})\end{array}$ & $\begin{array}{c}\text { FM }^{4} \\
\text { (d) }\end{array}$ & $\begin{array}{r}\text { MP5 } \\
(\%)\end{array}$ & $\begin{array}{l}\mathbf{A C H}^{6} \\
(1-5)\end{array}$ \\
\hline 8 & CMS 993013 & $9,57 *$ & 237 & 115 & 52 & $3,85 * *$ & $1,51 *$ \\
\hline 9 & CMS 993015 & $8,44^{*}$ & 218 & 120 & 52 & 1,58 & $1,49 *$ \\
\hline 19 & CMS 993035 & $8,08 * *$ & $247 *$ & 120 & 50 & $5,17 *$ & $1,54 *$ \\
\hline 2 & CMS 993001 & 7,95 & $251 *$ & $151^{*}$ & 53 & $4,28 *$ & $1,60 *$ \\
\hline 1 & CMS 973023 & 7,94 & 224 & 112 & $55^{*}$ & $3,95 *$ & $1,47^{*}$ \\
\hline 10 & CMS 993017 & 7,92 & 228 & $141^{*}$ & 50 & 0,62 & $1,85^{* *}$ \\
\hline 20 & CMS 993037 & 7,87 & 234 & 120 & $54 * *$ & 1,89 & 2,49 \\
\hline 18 & CMS 993033 & 7,86 & 233 & 121 & $55^{*}$ & $5,70 *$ & $1,49 *$ \\
\hline 4 & CMS 993005 & 7,78 & 211 & 127 & 52 & 1,38 & $1,73^{*}$ \\
\hline 5 & CMS 993007 & 7,34 & 219 & $136^{*}$ & 52 & 1,13 & $1,51 *$ \\
\hline 3 & CMS 993003 & 7,33 & $242 * *$ & $140 *$ & 53 & 1,48 & $1,86^{* *}$ \\
\hline 11 & CMS 993019 & 7,28 & 235 & $136 *$ & 53 & 1,45 & $1,88 * *$ \\
\hline 15 & CMS 993027 & 7,13 & 216 & $135^{*}$ & 51 & 1,41 & $1,73^{*}$ \\
\hline 22 & CMS 973049 & 7,02 & 214 & 109 & 51 & $3,51 * *$ & $1,60 *$ \\
\hline 13 & CMS 993023 & 7,00 & 229 & $129 * *$ & 52 & 2,13 & $1,68 *$ \\
\hline \multirow[t]{2}{*}{25} & H-520 & & & & & & \\
\hline & (Testigo) & 6,88 & 227 & $130 * *$ & 51 & 1,11 & $1,71 *$ \\
\hline 7 & CMS 993011 & 6,75 & $257 *$ & 126 & 52 & 0,00 & $1,71 *$ \\
\hline 6 & CMS 993009 & 6,70 & $249 *$ & $137 *$ & $56 *$ & $7,65^{*}$ & $1,61^{*}$ \\
\hline \multirow[t]{2}{*}{23} & CMS 933133 & & & & & & \\
\hline & $(\mathrm{RE})$ & 6,69 & 200 & 108 & 53 & 1,71 & $1,29 *$ \\
\hline 12 & CMS 993021 & 6,27 & $246 * *$ & 119 & 52 & 2,17 & 2,10 \\
\hline 16 & CMS 993029 & 5,90 & $262 *$ & $129 * *$ & 52 & $6,14 *$ & 2,47 \\
\hline 14 & CMS 993025 & 5,68 & 230 & 119 & 53 & $3,74 *$ & 2,45 \\
\hline 17 & CMS 993031 & 5,24 & $243 * *$ & $133 * *$ & $54 * *$ & $8,90 *$ & 2,87 \\
\hline 21 & CMS 993039 & 5,15 & 213 & 107 & 49 & $8,42 *$ & 2,86 \\
\hline \multirow[t]{5}{*}{24} & $\mathrm{H}-519 \mathrm{C}$ & & & & & & \\
\hline & (Testigo) & 4,49 & 229 & 107 & $54 * *$ & $4,40 *$ & 2,32 \\
\hline & Promedio & 7,05 & 232 & 125 & 52,51 & 3,35 & 1,87 \\
\hline & DMS 0,05 & 1,18 & 15,49 & 17,34 & 1,87 & 1,05 & 0,47 \\
\hline & DMS 0,01 & 1,59 & 20,76 & 23,24 & 2,50 & 1,41 & 0,63 \\
\hline
\end{tabular}

* = Significativo al 0,$05 ; *$ Significativo al 0,01 .

$\mathrm{RG}^{1}=$ rendimiento de grano; $\mathrm{AP}^{2}=$ altura de planta; $\mathrm{AM}^{3}=$ altura de mazorca; $\mathrm{FM}^{4}$ = floración masculina; $\mathrm{MP}^{5}=$ mazorcas podridas; $\mathrm{ACH}^{6}=$ calificación de la severidad de achaparramiento.

ba de DMS al 1\%, ocho híbridos resultaron estadísticamente superiores en altura de planta y 11 en altura de mazorca que los pone en desventaja con el resto. Se destaca que CMS 993035 que fue uno de los híbridos más rendidores tuvo un porte alto y que el testigo $\mathrm{H}$ 520 presentó la característica indeseable de posición de mazorca alta. En este sentido, lo deseable son los híbridos con menor altura de planta y posición intermedia de mazorca, ya que son más tolerantes al acame.

De acuerdo a la DMS, aunque seis genotipos resultaron estadísticamente superiores en el tiempo en que ocurrió su floración, su ciclo fue intermedio, ya que el máximo valor que se obtuvo fue de 56 días y sólo los híbridos CMS 993039, CMS 993017 y CMS 993035, cuyas floraciones medias ocurrieron a los 49 y 50 días se comportaron de manera precoz; en este sentido lo deseable es disponer de genotipos cuyas floraciones 
durante el ciclo primavera-verano se presenten antes de los 60 días.

Referente a la variable mazorcas podridas, 12 híbridos, incluyendo al testigo H-519 C, resultaron estadísticamente superiores con los más altos valores. En esta característica indeseable, los genotipos con mayor porcentaje fueron: CMS 993035, CMS 993033, CMS 993029, CMS 993009, CMS 993039 y CMS 993031. Hooker (1978) señala que la herencia de la resistencia a las pudriciones de mazorca es poligénica, de acción génica aditiva y semidominante. De modo que los métodos de mejoramiento por retrocruza y selección recurrente reciproca, pueden ser eficaces en el mejoramiento de esta característica (Betanzos 2001). En general, este carácter no tuvo un efecto negativo muy marcado en el rendimiento de grano en esta localidad $(r=-0,402 *)$.

En cuanto a la variable severidad del achaparramiento, de acuerdo a la DMS, 18 materiales resultaron sobresalientes con menores daños ocasionados por la enfermedad bajo condiciones de infestación natural del vector y cuyas calificaciones fueron de 1,88 a 1,29. Para el caso de los testigos regionales, el híbrido H-519 C fue el más afectado. Pese a que en general, la severidad de la enfermedad tuvo un grado máximo de tres en algunos genotipos, se encontró una correlación negativa y altamente significativa $(r=-0,694 * *)$ que indicó que el rendimiento disminuyó a medida que la severidad de la enfermedad fue mayor.

Estudios realizados en el área tropical de este estado con líneas e híbridos de maíz bajo condiciones de infestación artificial de Dalbulus maydis, han revelado que existen diferencias en respuesta a la presencia del achaparramiento (Hidalgo et al. 1998; Cano et al. 2000). Considerando que la herencia de la resistencia al achaparramiento no es dominante, si no que es un carácter controlado por pocos pares de genes aditivos y no epistáticos, un programa de selección recurrente puede ser adecuado para transferir resistencia a materiales susceptibles (Ortíz et al. 1992). Por otro lado, los materiales tolerantes representan una base de germoplasma para la derivación de líneas en los programas de mejoramiento genético (Cano et al. 2000).

En la localidad de San Andrés Tuxtla (Cuadro 2), 10 híbridos resultaron sobresalientes (DMS al 1\%), de los cuales CMS 973023 y CMS 993033 lograron rendimientos superiores a las 7 t/ha. Los más bajos rendimientos se obtuvieron con los testigos H-520 y H-519 C. Con relación a la variable altura de planta y mazorca, siete y nueve genotipos, respectivamente, resultaron estadísticamente más altos. CMS 933133 (RE) con 196 cm y CMS 993027 con 202 cm, fueron los híbridos con
Cuadro 2. Rendimiento y características agronómicas de híbridos de maíz en San Andrés Tuxtla, Veracruz, México. 2002.

\begin{tabular}{|c|c|c|c|c|c|c|}
\hline No. & Genotipo & $\begin{array}{c}\mathbf{R G}^{\mathbf{1}} \\
(\mathbf{t} / \mathbf{h a})\end{array}$ & $\begin{array}{l}\mathbf{A P}^{2} \\
(\mathbf{c m})\end{array}$ & $\begin{array}{l}\mathrm{AM}^{3} \\
(\mathrm{~cm})\end{array}$ & $\begin{array}{c}\mathrm{FM}^{4} \\
\text { (d) }\end{array}$ & $\begin{array}{l}\text { MP5 } \\
(\%)\end{array}$ \\
\hline 1 & CMS 973023 & $7,52^{*}$ & 218 & 120 & 54 & 3,20 \\
\hline 18 & CMS 993033 & $7,29 *$ & 226 & $126 * *$ & $56^{* *}$ & 2,48 \\
\hline 7 & CMS 993011 & $6,9^{*}$ & $254 *$ & $129 * *$ & 54 & 3,09 \\
\hline 13 & CMS 993023 & $6,68 *$ & 232 & 121 & 54 & 3,18 \\
\hline 19 & CMS 993035 & $6,64 *$ & $248 *$ & 125 & 54 & 1,52 \\
\hline 3 & CMS 993003 & $6,47 * *$ & 235 & $139 *$ & $55^{* *}$ & 0,00 \\
\hline 11 & CMS 993019 & $6,42 * *$ & 223 & $135^{*}$ & $56 * *$ & $6,17 *$ \\
\hline 2 & CMS 993001 & $6,36^{* *}$ & $249^{*}$ & $133^{*}$ & $55^{* *}$ & 3,40 \\
\hline 4 & CMS 993005 & $6,33 * *$ & 219 & 125 & $55^{* *}$ & 1,67 \\
\hline 5 & CMS 993007 & $6,26 * *$ & 218 & 124 & $56 * *$ & 2,96 \\
\hline 17 & CMS 993031 & 6,17 & $246^{*}$ & $126 * *$ & $55^{* *}$ & 2,55 \\
\hline 20 & CMS 993037 & 6,13 & 218 & 110 & $56 * *$ & 1,71 \\
\hline 21 & CMS 993039 & 6,08 & 209 & 116 & 53 & 3,93 \\
\hline 10 & CMS 993017 & 5,97 & 216 & 100 & 54 & 1,46 \\
\hline 12 & CMS 993021 & 5,89 & 230 & 121 & 54 & 2,56 \\
\hline 22 & CMS 973049 & 5,85 & 215 & 96 & 51 & 2,68 \\
\hline 6 & CMS 993009 & 5,70 & $238 * *$ & $136^{*}$ & $56^{* *}$ & $8,57 *$ \\
\hline 8 & CMS 993013 & 5,59 & $240 * *$ & $127 * *$ & 54 & $4,26 * *$ \\
\hline 15 & CMS 993027 & 5,55 & 202 & 109 & 53 & $5,45^{* *}$ \\
\hline 9 & CMS 993015 & 5,36 & 207 & 107 & 53 & $4,50 * *$ \\
\hline 14 & CMS 993025 & 5,34 & 224 & 121 & $55^{* *}$ & $6,00^{*}$ \\
\hline 23 & CMS 933133 (RE) & 5,27 & 196 & 111 & $55^{* *}$ & $6,04 * *$ \\
\hline 16 & CMS 993029 & 5,08 & $253^{*}$ & $130 *$ & $55^{* *}$ & $7,63^{*}$ \\
\hline 25 & H-520 (Testigo) & 4,53 & 218 & 112 & 53 & $10,31 *$ \\
\hline \multirow[t]{4}{*}{24} & H-519 (Testigo) & 3,95 & 219 & 113 & 54 & $11,6^{*}$ \\
\hline & Promedio & 5,97 & 226,04 & 120,44 & 54,37 & 4,28 \\
\hline & DMS 0,05 & 0,94 & 12,94 & 9,95 & 1,42 & 0,99 \\
\hline & DMS 0,01 & 1,26 & 17,34 & 13,33 & 1,91 & 1,32 \\
\hline
\end{tabular}

$*=$ Significativo al 0,$05 ; * *$ Significativo al 0,01 .

$\mathrm{RG}^{1}=$ rendimiento de grano; $\mathrm{AP}^{2}=$ altura de planta; $\mathrm{AM}^{3}=$ altura de mazorca; $\mathrm{FM}^{4}=$ floración masculina; $\mathrm{MP}^{5}=$ mazorcas podridas.

menor altura de planta, no obstante, presentaron bajo rendimiento. Por su parte, los dos genotipos de mayor rendimiento presentaron altura de planta y de mazorca intermedia.

En la evaluación de genotipos es importante considerar además del rendimiento de grano, la altura de planta y mazorca, ya que son variables que presentan alta correlación con el acame de plantas (Sierra 2002). Por lo que, estos resultados son satisfactorios, ya que en la zona de San Andrés Tuxtla se presentan vientos fuertes que pueden provocar el acame de plantas.

Las floraciones ocurridas en los genotipos fueron intermedias, con valores que variaron de 51 a 56 días. En cuanto a la variable mazorcas podridas, un grupo de 10 genotipos entre los que se incluyen los testigos, resultaron estadísticamente con mayor grado de pudrición y fueron además los de menor rendimiento. El coeficiente de correlación de esta variable con el 
rendimiento de grano fue de $\mathrm{r}=-0,758^{* *}$, el cual sugiere alto grado de asociación inversamente proporcional entre ambas variables, por lo que en general, el porcentaje de pudrición de mazorcas tuvo un efecto negativo y altamente significativo en el rendimiento de grano en esta localidad. Esta característica evaluada, es de gran importancia, ya que en el área de San Andrés Tuxtla, la alta humedad relativa, favorece la presencia de hongos causantes de pudriciones de mazorca, por lo que es conveniente que los genotipos tengan buena protección por el totomoxtle (Ireta 1988; Rueda 1999).

En el CIMMYT, después de 16 ciclos de selección recurrente de medios hermanos, se han logrado avances importantes en el mejoramiento de la resistencia a la pudrición de mazorca causada por Fusarium monilifor$m e$, así como en el incremento en el rendimiento de poblaciones tropicales (De León y Pandey 1989). A su vez, Betanzos (2001) en un estudio conducido en la depresión central de Chiapas, encontró que los genotipos de CIMMYT CMS943053 y CMS943067 registraron los más altos rendimientos y la mayor resistencia a la pudrición de mazorca.

El análisis combinado para rendimiento de grano detectó alta significancia para Localidades (L), Híbridos $(\mathrm{H})$ y la interacción $\mathrm{H}$ x L, lo que señala que existieron diferencias sobre el potencial productivo por efecto de las localidades de prueba, respecto a la capacidad productiva de los genotipos y en el comportamiento de algunos de estos híbridos, que fue diferente de una localidad a otra. El coeficiente de variación fue de $9,88 \%$.

Los mayores rendimientos por localidad se consiguieron en Tlalixcoyan, que presentó un promedio de 7,05 t/ha, superior en 1,08 t/ha a la localidad de San Andrés Tuxtla (Cuadro 3). En el factor Híbridos, un grupo de 14 genotipos resultaron estadísticamente superiores, entre los cuales, siete obtuvieron rendimientos superiores a 7 t/ha, sin embargo, en este grupo selecto, sólo CMS 973023 y CMS 993033 lograron mantener alto rendimiento en ambas localidades. CMS 993013 disminuyó su rendimiento en 3,98 t/ha al establecerse en San Andrés Tuxtla, mientras que los otros cuatro lo hicieron en alrededor de 1,5 t/ha.

De los resultados obtenidos se concluye que por su alto rendimiento, buen comportamiento agronómico y de reacción al achaparramiento, los mejores híbridos para Tlalixcoyan fueron: CMS 993013 y CMS 993015. Para San Andrés Tuxtla, CMS 973023 y CMS 993033 de porte y ciclo intermedio fueron los híbridos más rendidores y con bajos porcentajes de mazorcas podridas. Los mejores rendimientos promedio se obtuvieron con
Cuadro 3. Rendimiento promedio de híbridos de maíz a través de las localidades de evaluación. Veracruz, México. 2002.

\begin{tabular}{rlccc}
\hline No. & Genotipo & $\begin{array}{c}\text { Tlalixcoyan } \\
(\mathbf{t} / \mathbf{h a})\end{array}$ & $\begin{array}{c}\text { S. Andrés T. } \\
\text { (t/ha) }\end{array}$ & $\begin{array}{c}\text { Promedio } \\
\text { (t/ha) }\end{array}$ \\
\hline & & & & \\
1 & CMS 973023 & 7,94 & 7,52 & $7,73^{*}$ \\
8 & CMS 993013 & 9,57 & 5,59 & $7,58^{*}$ \\
18 & CMS 993033 & 7,86 & 7,29 & $7,57^{*}$ \\
19 & CMS 993035 & 8,08 & 6,64 & $7,36^{*}$ \\
2 & CMS 993001 & 7,95 & 6,36 & $7,15^{*}$ \\
4 & CMS 993005 & 7,78 & 6,33 & $7,05^{*}$ \\
20 & CMS 993037 & 7,87 & 6,13 & $7,00^{*}$ \\
10 & CMS 993017 & 7,92 & 5,97 & $6,94^{*}$ \\
9 & CMS 993015 & 8,44 & 5,36 & $6,90^{*}$ \\
3 & CMS 993003 & 7,33 & 6,47 & $6,90^{*}$ \\
11 & CMS 993019 & 7,28 & 6,42 & $6,85^{*}$ \\
13 & CMS 993023 & 7,00 & 6,68 & $6,84^{*}$ \\
7 & CMS 993011 & 6,75 & 6,90 & $6,82^{*}$ \\
5 & CMS 993007 & 7,34 & 6,26 & $6,80^{*}$ \\
22 & CMS 973049 & 7,02 & 5,85 & 6,43 \\
15 & CMS 993027 & 7,13 & 5,55 & 6,34 \\
6 & CMS 993009 & 6,70 & 5,70 & 6,20 \\
12 & CMS 993021 & 6,27 & 5,89 & 6,08 \\
23 & CMS 933133 (RE) & 6,69 & 5,27 & 5,98 \\
17 & CMS 993031 & 5,24 & 6,17 & 5,70 \\
25 & H,520 (Testigo) & 6,88 & 4,53 & 5,70 \\
21 & CMS 993039 & 5,15 & 6,08 & 5,61 \\
14 & CMS 993025 & 5,68 & 5,34 & 5,51 \\
16 & CMS 993029 & 5,90 & 5,08 & 5,49 \\
24 & H-519 C (Testigo) & 4,49 & 3,95 & 4,22 \\
\hline & & & & \\
& Promedio & $7,05 * *$ & 5,97 & 6,51 \\
& DMS 0,05 & 0,21 & 0,21 & 0,74 \\
& DMS 0,01 & 0,28 & 0,28 & 0,98 \\
\hline & & & & \\
\hline
\end{tabular}

$*=$ Significativo al 0,$05 ; * *$ Significativo al 0,01 .

CMS 973023, CMS 993013 y CMS 993033, aunque sólo el primero y el tercero mantuvieron alto rendimiento en ambas localidades. La severidad del achaparramiento en Tlalixcoyan y el porcentaje de pudrición de mazorcas manifestado en San Andrés Tuxtla afectaron significativamente el rendimiento de grano de maíz.

\section{LITERATURA CITADA}

BARRETO, H. J. 1999. Alpha programa para crear y analizar diseños Alpha $(0,1)$ Látice. In: El programa de maíz del CIMMYT, manual del usuario para Fieldbook 5.1/7.1 y Alpha México, D. F. p. 38-48.

BETANZOS, M. E. 2001. Variedades de maíz resistentes, una opción para reducir la pudrición de mazorca en Chiapas, México. Agricultura Técnica en México 27 (1): 57 - 67.

CEPEDA, M.; OROZCO, G. 1989. Enfermedades del maíz en Michoacán. In: D. Teliz; C. Mendoza; S. Romero 
(eds.). Memoria del XVI Congreso Nacional de Fitopatología. Montecillos, Estado de México. p. 1.

CANO, O.; SIERRA, M.; JEFFERS, D.; TOSQUY, O. H.; PALAFOX, A.; PRECIADO, R. E. 2000. Respuesta de híbridos de maíz QPM y normales a infestación con (Dalbulus maydis) vector del achaparramiento del maíz. In: H. Barradas (ed.). Memoria de la Décima Tercera Reunión Científica, Tecnológica, Forestal y Agropecuaria del estado de Veracruz. Veracruz, Ver., México. s/p (Memoria en CD).

DE LEÓN, C.; PANDEY, S. 1989. Improvement of resistence to ear and stalk rots and agronomic traits in tropical maize gene pools. Crop Sci. 29: 12 -17.

GARCÍA, E. 1981. Modificaciones al sistema de clasificación climática de Köppen. Instituto de Geografía de la UNAM. México. $3^{\text {a }}$. Ed. 252 p.

HENRÍQUEZ, P.; JEFFERS, D. 1997. El achaparramiento del maíz: Patógenos, síntomas y diagnóstico. In: J. Bolaños (ed.). Síntesis de resultados experimentales del Programa Regional de Maíz para Centro América y el Caribe1993-1995. CIMMYT - PRM, Guatemala 5: 283-290.

HIDALGO, H., JEFFERS, D.; CASTAÑÓN, G.; RODRÍGUEZ, F. 1998. Resistencia al achaparramiento del maíz mediante infestaciones de Dalbulus maydis en maíz. Agronomía Mesoamericana. 9 (2): 119-124.

HOOKER, A. L. 1978.Genetics of disease resistance in maize. In: Walden, D. B. (ed.). Maize breeding and genetics. John Wiley and Sons. New York. U. S. A. p. 319- 332.

INSTITUTO NACIONAL DE ESTADÍSTICA, GEOGRAFÍA E INFORMÁTICA (INEGI). 2002. Anuario estadístico del estado de Veracruz. Tomo II. Aguascalientes, Ags., México. p. 569.

IRETA, M. J. 1988. Pudriciones de la mazorca. In: D. Téliz (ed.). Enfermedades del maíz, frijol, trigo y papa. Centro de Fitopatología. Colegio de Postgraduados. p. 12 - 15.

LÓPEZ, C. J. 1998. Interpretación de los resultados de los análisis químicos de suelos agrícolas. Colegio de postgraduados. Instituto de Fitosanidad. Manlio Fabio Altamirano, Veracruz, México. 45 p.

MORRIS, L.; LÓPEZ, M. A. 2000. Impactos del mejoramiento genético de maíz en América Latina. 1966 - 1997. México, D. F. CIMMYT. 45 p.

ORTÍZ, J.; NAVARRO, F.; CELADO, R. 1992. Mejoramiento genético del ciclo 4 de la población 36 para resisten- cia al achaparramiento de maíz. In: J. Bolaños (ed.). Síntesis de resultados experimentales del Programa Regional de Maíz para Centro América y el Caribe. CIMMYT - PRM, Guatemala. p. 76-78.

PALAFOX, A.; SIERRA, M.; TURRENT, A.; TOSQUY, O. H.; BARRÓN, S.; CANO, O. 2001. Respuesta de híbridos de maíz normales y de alta calidad de proteína a niveles de fertilización y densidad. In: H. Barradas (ed.). Memoria de la Décima Cuarta Reunión Científica, Tecnológica, Forestal y Agropecuaria del estado de Veracruz. Veracruz, Ver., México. s/p (Memoria en CD).

REYES, P. 1982. Bioestadística aplicada. Trillas. México, D. F. p.163.

REYES, P. 1990. El maíz y su cultivo. AGT Editor S: A. p. 103-153.

ROCHA, M. A. 1987. Descripción de las enfermedades del maíz (Zea mays L.) en el trópico. In: E. Becerra; H. Lozoya (eds.). Taller de Fitopatología Tropical. Centro de Enseñanza, Investigación y Capacitación para el Desarrollo Agropecuario, Forestal y Acuicola del Sureste (CEICADES). Colegio de Postgraduados. Chapingo, México. p. 425 - 449.

RUEDA, P. 1999. Evaluación agronómica de genotipos de maíz en el área de influencia de San Andrés Tuxtla, Ver. Tesis Profesional de Ingeniero Agrónomo. Facultad de Ciencias Agrícolas. U. V., Córdoba, Veracruz, México. 60 p.

SIERRA, M. 2002. Uso de probadores en la selección de líneas para formar híbridos de maíz (Zea mays L.). Tesis de Doctorado en Ciencias. Tecomán, Colima, México. $177 \mathrm{p}$.

TINOCO, C. A.; RODRÍGUEZ, F. A.; SANDOVAL, J. A.; BARRÓN, S.; PALAFOX, A.; ESQUEDA, V. A.; SIERRA, M.; ROMERO, J. 2002. Manual de producción de maíz para los estados de Veracruz y Tabasco. Libro Técnico Núm. 9. INIFAP. CIRGOC. Campo Experimental Papaloapan. Veracruz, México. 113 p.

TOSQUY, O. H.; VÁSQUEZ, A.; LÓPEZ, V. O. 2001. Rendimiento de grano y principales características agronómicas de cultivares de maíz en el sur de Veracruz, México. In: R. Araya; N. Chaves (comp.). Resúmenes de la XLVII Reunión Anual del PCCMCA. San José, Costa Rica. p.57.

UNIVERSIDAD AUTÓNOMA CHAPINGO. 1982. Inventario de áreas erosionadas, rangos de pendiente y unidades de suelo del estado de Veracruz. Chapingo, México. $139 \mathrm{p}$. 